\title{
28 Research Square \\ Predictive values of multiple serum biomarkers in women with suspected preeclampsia: a prospective study
}

\section{Jing Wang}

Capital Medical University Beijing Obstetrics and Gynecology Hospital Honghai Hu

Guangzhou Kangrun Biotech Co. Ltd.

Xiaowei Liu

Capital Medical University Beijing Obstetrics and Gynecology Hospital

Shenglong Zhao

Capital Medical University Beijing Obstetrics and Gynecology Hospital

\section{Yuanyuan Zheng}

Capital Medical University Beijing Obstetrics and Gynecology Hospital

\section{Zhaoxia Jia}

Capital Medical University Beijing Obstetrics and Gynecology Hospital

\section{Lu Chen}

Capital Medical University Beijing Obstetrics and Gynecology Hospital

Chunhong Zhang

Capital Medical University Beijing Obstetrics and Gynecology Hospital

\section{Xin Xie}

Capital Medical University Beijing Obstetrics and Gynecology Hospital Junhui Zhong

Sysmex Shanghai Ltd.

\section{Ying Dong}

Capital Medical University Beijing Obstetrics and Gynecology Hospital Jingrui Liu

Capital Medical University Beijing Obstetrics and Gynecology Hospital

Yifan Lu

Capital Medical University Beijing Obstetrics and Gynecology Hospital

\section{Zhen Zhao}

Weill Cornell Medine

\section{Yanhong Zhai}

Capital Medical University Beijing Obstetrics and Gynecology Hospital Juan Zhao 
Capital Medical University Beijing Obstetrics and Gynecology Hospital

\section{Zheng Cao ( $\square$ zhengcao2011@hotmail.com )}

Capital Medical University Beijing Obstetrics and Gynecology Hospital https://orcid.org/0000-00020968-1864

\section{Research article}

Keywords: preeclampsia, prediction, serum biomarker, prospective, cohort

Posted Date: September 10th, 2020

DOI: https://doi.org/10.21203/rs.3.rs-33584/v3

License: (9) This work is licensed under a Creative Commons Attribution 4.0 International License. Read Full License 


\section{Abstract}

Background: Preeclampsia is a common obstetric multisystem disorder causing maternal and fetal morbidity and mortality; it's been shown that the prediction improves preeclampsia outcomes in pregnancy. However, the current serum biomarkers had low clinical application values and still lack validation studies. Here we aimed to evaluate the preeclampsia prediction values of a series of serum biomarkers in Chinese pregnant women of $>20$ weeks of gestation.

Methods: Singleton pregnant women with preeclampsia-related clinical and/or laboratory presentations were recruited and had blood drawn at their first visits. The prospective cohort was further divided into preeclampsia-positive and preeclampsia-negative groups based on the follow-up results. The following markers were tested using the collected serum samples: soluble fms-like tyrosine kinase-1 (sFlt-1); placental growth factor (PIGF); thrombomodulin (TM); tissue plasminogen activator inhibitor complex (tPAl-C); compliment factors $\mathrm{C} 1 \mathrm{q}, \mathrm{B}$, and $\mathrm{H}$; glycosylated fibronectin (GlyFn); pregnancy-associated plasma protein-A2 (PAPP-A2); blood urea nitrogen (BUN); creatinine (Cre); uric acid (UA); and cystatin C (Cysc).

Results: A total of 196 women with suspected preeclampsia were recruited with follow-up medical records. Twenty-five percent $(n=49)$ of the recruited subjects developed preeclampsia before delivery, and $75 \%$ remained preeclampsia-negative $(n=147)$. The serum levels of sFIt-1, BUN, Cre, UA, Cysc and PAPPA2 were significantly elevated, and the PIGF level was significantly decreased in the preeclampsiapositive patients. In the receiver operating characteristics (ROC) analyses, the area under the curves were listed in the order of decreasing values: 0.73 (UA), 0.67 (sFlt-1/PIGF), 0.66 (Cysc), 0.65 (GlyFn/PIGF), 0.64 (PAPP-A2/PIGF), 0.63 (BUN), 0.63 (Cre), and 0.60 (PAPP-A2). With the cut-off values obtained from the $\mathrm{ROC}$ analyses, the positive predictive values of these serum markers were between $33.1 \%$ and $58.5 \%$, and the negative predictive values were between $80.9 \%$ and $89.5 \%$.

Conclusions: Although several serum markers were found to be significantly changed with current prospective cohort, their limited predictive values in preeclampsia development posed potential barrier in clinical implementation. Further studies with larger cohort are warranted to further reveal the clinical utilities of the serum markers in preeclampsia prediction.

\section{Introduction}

Preeclampsia, one of the most common complications during pregnancy, is estimated to have an incidence rate of $2-8 \%$ worldwide (1) and can lead to serious maternal or fetal morbidity and mortality if not managed properly (2). Extensive studies have been performed to reveal the clinical value of preeclampsia prediction (3), of which three major beneficial effects may be concluded: identifying highrisk patients who require close monitoring to decrease potential complications, reducing the necessity of antenatal care of low-risk populations, and promoting the development of early therapeutic interventions of preeclampsia (4). 
According to a systematic review published in 2019 about preeclampsia prediction models (5), most previous studies (87\%) have conducted risk assessments during the first trimester of pregnancy. In first trimester preeclampsia screening, the prediction model is recommended to combine maternal background risk factors, imaging tests and serum biomarkers to increase sensitivity and reduce the false detection rate (5). Due to relatively low positive predictive values (PPV) (8-33\%) during first trimester screening for preeclampsia (6), false-positive patients who do not develop preeclampsia may be exposed to unnecessary tests and prophylactic interventions with no benefit.

Recently, some researchers evaluated preeclampsia predictive markers in patient groups $>20$ gestational weeks and identified high-risk factors or clinical/laboratory signs of preeclampsia $(7,8)$. This type of testing scenario with patients suspected of preeclampsia development has been proven effective, especially in the studies on soluble fms-like tyrosine kinase-1 (sFlt-1) and placental growth factor (PIGF) $(7,9)$. For example, a sFIt-1/PIGF ratio of 38 was found to effectively exclude preeclampsia with a negative predictive value (NPV) of $99.3 \%$ (7); however, a similar prospective study with Chinese pregnant women has yet to be published. Interestingly, a recent longitudinal study conducted in Singapore suggested significant differences in the PIGF and sFlt-1 concentrations during pregnancy between different Asian ethnicities (Chinese, Malay, and Indian) (10). In a retrospective study with 118 Chinese singleton pregnancies who had been diagnosed with preeclampsia, the sFlt-1/PIGF ratio was shown to be an efficient marker in differentiating preeclampsia and predicting the timing of delivery for preeclampsia pregnancies (11).

In addition to sFIt-1/PIGF, a series of other serum biomarkers have been shown to be associated with the occurrence or outcomes of preeclampsia. For instance, the maternal pregnancy-associated plasma protein-A2 (PAPP-A2) serum concentration was found to be upregulated in preeclampsia patients, resulting in local activation of insulin-like growth factor (IGF) signaling pathways (12). This finding implied that PAPP-A2 may be up-regulated in preeclampsia to compensate for IGF binding protein 5mediated pathway (12). The maternal serum glycosylated fibronectin (GlyFn) was reported to be elevated in all three trimesters of preeclampsia patients; the test was further recommended as a point-of-care biomarker to quickly determine risk for preeclampsia and for poor maternal and fetal outcomes among preeclamptic patients (13). In uteroplacental thrombosis, which is one of the major mechanisms of preeclampsia, several thrombotic and fibrinolytic factors including circulating soluble thrombomodulin (TM) and tissue plasminogen activator (tPA) were found to be elevated in PE and correlated with the severity of proteinuria $(14,15)$. The relative changes of these coagulation factors reflected endothelial disturbance in preeclampsia and they were recommended for future evaluation as potential risk biomarkers $(14,15)$. The dysregulation of complement pathways also contributes to the development of preeclampsia. The differential expression of complement factors $\mathrm{C} 1 \mathrm{q}, \mathrm{B}$ and $\mathrm{H}$ were found in specific trimesters of severe preeclampsia patients, suggesting promising values as diagnostic markers for severe preeclampsia (16). The presence of proteinuria, which is a hallmark in preeclampsia, indicates that renal deficiency contributes significantly to the pathogenesis of preeclampsia (17). The renal function markers, such as uric acid (UA), blood urea nitrogen (BUN), creatinine (Cre), cystatin C (Cysc), have been found to 
be disturbed in preeclampsia patients (17) and their performance in predicting preeclampsia after 20 weeks of gestation still lacks validation studies.

In summary, even though the panel of serum markers described above have been studied in a broad context of preeclampsia, whether or not these biomarkers will add value in preeclampsia prediction remains largely unknown. In this work, we aimed to evaluate the predictive values of: [1] the known markers of sFlt-1 and PIGF, and [2] PAPP-A2, GlyFn, TM, tissue plasminogen activator inhibitor complex (tPAl-C), compliment factors $\mathrm{C} 1 \mathrm{q}, \mathrm{B}$, and $\mathrm{H}$, and renal function markers including $\mathrm{UA}, \mathrm{BUN}$, Cre, and Cysc, in a prospective study with Chinese pregnant women who were suspected to develop preeclampsia.

\section{Methods}

\section{Subjects}

The enrollment criteria for women with suspected preeclampsia are described as follows. The recruited singleton pregnant women were at least 18 years old and between 20-36 gestational weeks (GWs), as pregnancies of $>36 \mathrm{GWs}$ are likely to be subjected to delivery of fetus if preeclampsia is diagnosed or the blood pressure (BP) is severely elevated. In addition, one of the following recruiting criteria had to be met for patient enrollment: new onset of hypertension (systolic BP $>120$ and $<160 \mathrm{mmHg}$ and/or diastolic BP $>80$ and $<110 \mathrm{mmHg}$ ) or proteinuria ( $\geq 2+$ by dipstick); aggravation of preexisting hypertension or proteinuria; or persistent symptoms of upper abdominal pain, edema, headache, visual impairment, abnormal weight gain $\left(>1 \mathrm{~kg} /\right.$ week), decreased platelets $\left(<150 * 10^{9} / \mathrm{L}\right)$, elevated liver transaminase (alanine transferase $>55 \mathrm{U} / \mathrm{L}$ or aspartate transaminase $>34 \mathrm{U} / \mathrm{L}$ ), fetal growth restriction (estimated fetal weight or abdominal circumference $<10$ th percentile according to the charts routinely used by Obstetric Department at our institute), abnormal uterine ultrasound perfusion during mid-pregnancy, or uterine artery flow notching. The subjects meeting one of the following conditions were excluded: confirmed diagnosis of preeclampsia or Hemolysis Elevated Liver enzymes and Low Platelets (HELLP) syndrome or anti-hypertensive treatment during this pregnancy. The recruited pregnant subjects had their blood drawn at their first visits to Beijing Obstetrics and Gynecology Hospital, with follow-up for the presence ("preeclampsia-positive" group) or absence ("preeclampsia-negative" group) of preeclampsia until delivery.

The preeclampsia diagnosis was determined with the diagnostic criteria proposed by the 2019 ACOG Practice Bulletin (6), in which preeclampsia was defined as gestational hypertension (systolic/diastolic blood pressure $\geq 140 / 90 \mathrm{mmHg}$ ) in previously normotensive women accompanied by proteinuria (urine protein $\geq 300 \mathrm{mg} / 24$ hours) or end-organ damage after 20 weeks of gestation.

\section{Serum samples, reagents and methods}

The maternal blood from each participant $(3 \mathrm{ml})$ was drawn when they were enrolled and left to clot for $30 \mathrm{~min}$ followed by centrifugation for $10 \mathrm{~min}$ at $2300 \mathrm{~g}$. The serum aliquots $(1 \mathrm{ml})$ were separated and stored at $-80^{\circ} \mathrm{C}$ until being tested. 
The maternal levels of sFlt-1 (Cat No. YZB/GER5424-2014, Germany, Roche Diagnostics) and PIGF (Cat No. YZB/GER5425-2014, Germany, Roche Diagnostics) were measured on the fully automated electrochemiluminescence immunoassay platform COBAS e411 (Germany, Roche Diagnostics). Maternal serum PAPP-A2 (Cat No. AL109, USA, AnshLabs) and GlyFn (Cat No. AL160, USA, AnshLabs) levels were determined with single measurement by the enzyme-linked immunosorbent assay (ELISA) according to the manufacturer's instructions. The total coefficients of variation (CVs) for PAPP-A2 and GlyFn were $4.1 \%-4.7 \%$ and $3.2-3.4 \%$, respectively. The ELISA standard operation protocol was performed as previously described $(5,18)$. The serum TM $\left(\mathrm{HISCL}^{\circledR} \mathrm{TM}\right.$ Assay Kit, Japan, Sysmex) and tPAl-C $\left(\mathrm{HISCL}^{\circledR}\right.$ tPAI-C Assay Kit, Japan, Sysmex) levels were determined by the fully automated HISCL-5000 Chemiluminescence Analyzer (Japan, Sysmex). The complement factors C1q (Cat No. 20170922, China, Shanghai Beijia Biochemical Reagent), B (Cat No. 20020803, China, Shanghai Beijia Biochemical Reagent) and $\mathrm{H}$ (Cat No. 20183020, China, Shanghai Beijia Biochemical Reagent) levels were measured using a fully automated ARCHITECT ci16200 Integrated System Chemistry/Immunology Analyzer (USA, Abbott). The renal function tests including UA (Cat No. 3P39-21, USA, Abbott), BUN (Cat No. 7D75-21, USA, Abbott), Cre (Cat No. 8L24-31, USA, Abbott) and Cysc (Cystatin C Assay Kit, China, Beijing Jiuqiang Biotech) were also performed on the ARCHITECT ci16200 Analyzer (USA, Abbott).

\section{Statistical analysis}

Data analysis was performed using statistical software SPSS 23.0. The Kolmogorov-Smirnov test was used to evaluate the normality of the data distribution. Numerical values were expressed as the mean and standard deviation (SD) for variables with normal distribution and as the median and percentiles for nonnormally distributed data. Comparisons between the two groups were performed using the t-test (for normal distribution) or Mann-Whitney-U test (for nonnormal distribution). Categorical variables were expressed as frequencies and proportion; comparisons between the two groups were tested by Chi-square test. The receiver operating characteristics (ROC) curve was used to analyze the predictive values of the markers for preeclampsia. The comparison of before and after adjusted area under curves (AUCs) was assessed using the algorithm developed by DeLong (19). The adjusted factors included age, prepregnancy BMI, parity, and underlying chronic diseases in the ROC analyses. Sensitivity, specificity and cut-off values were reported when Youden's index was at the maximum or specificity was fixed at $90 \%$. A binary Logistic regression analysis was performed including age, prepregnancy BMI, parity, underlying chronic diseases and each of the markers. All statistical tests were two-sided and $p<0.05$ was considered statistically significant.

\section{Results}

A flowchart depicting patient recruitment and the study design is presented in Figure 1. From January 2018 to March 2019, a total of 200 subjects with preeclampsia related clinical and/or laboratory presentations were recruited, including 4 patients who were lost to follow-up. Of the remaining 196 patients, 25\% (49/196) (preeclampsia-positive group) developed preeclampsia before delivery, and 75\% (147/196) (preeclampsia-negative group) maintained preeclampsia negative for remainder of the 
pregnancy. The demographic data of all the recruited subjects are available in Supplementary Table 1. As shown in Supplementary Table 2, no significant difference was found between the preeclampsia positive and negative groups for the main symptoms identified in the enrolled patients (new onset of hypertension or aggravation of preexisting hypertension, new onset of proteinuria or aggravation of preexisting proteinuria, edema and fetal growth restriction).

The collected serum samples of the preeclampsia-positive $(n=49)$ and preeclampsia-negative $(n=147)$ patients were subjected to the following serum marker measurements: sFIt-1; PIGF; PAPP-A2; GlyFn; TM; tPAI-C; compliment factors C1q, B, and H; UA; BUN; Cre; and Cysc. As summarized in Table 1, except for underlying chronic diseases, no significant difference was found in maternal age, prepregnancy BMI, blood sampling gestational weeks (GW), gravidity, or parity between the two groups; however, patients with underlying diseases (hypertension being the most prominent) that are associated with preeclampsia development were more likely to develop preeclampsia during pregnancy $(p=0.010$, Table 1$)$. On average, the time interval from serum collection to preeclampsia occurrence was 7 weeks in the preeclampsiapositive patients (Table 1).

To evaluate the preeclampsia predictive values of the selected markers in present study, we first compared their serum concentrations that were determined using our laboratory devices and platforms. As shown in Table 2, a panel of analytes representing various biological functions were found to be significantly elevated in the patients who developed preeclampsia later in pregnancy compared to the preeclampsia-negative group, including sFlt-1 ( $p=0.007), B U N(p=0.009)$, Cre $(p=0.006), U A(p<0.001)$, Cysc $(p=0.001)$ and PAPP-A2 $(p=0.032)$. The PIGF level $(p=0.004)$ was the only marker that was significantly decreased in the preeclampsia-positive patients, resulting in more profoundly increased calculated ratios, including sFlt-1/PIGF ( $p<0.001)$, GlyFn/PIGF ( $p=0.002)$, and PAPP-A2/PIGF $(p=0.003)$. By contrast, the hemostatic factors (TM and tPAI-C) and the compliment factors (C1q, B, and H) were not significantly different between the preeclampsia negative and positive groups. The significantly changed serum markers either from direct laboratory measurements or from the ratio calculations were then subjected to ROC analyses. As shown in Figure 2 and Table 3, the AUCs were listed in the order of decreasing values: 0.73 (UA), 0.67 (sFIt-1/PIGF), 0.66 (Cysc), 0.65 (GlyFn/PIGF), 0.64 (PIGF), 0.64 (PAPPA2/PIGF), 0.63 (sFlt-1), 0.63 (BUN), 0.63 (Cre), and 0.60 (PAPP-A2). After adjusted for age, prepregnancy $\mathrm{BMI}$, parity and underlying chronic diseases, the improvement of the prediction power of each serum marker was insignificant ( $p>$ or $=0.05$ ) (Table 3$)$.

The serum marker measurements were subsequently analyzed by binary logistic regression analysis, with age, prepregnancy BMI, parity and underlying chronic diseases as covariates. The logistic regression analysis showed that all the listed serum markers were independent risk factors $(p<0.05)$ for preeclampsia development, with Cysc, BUN and PAPP-2/PIGF having the highest OR values (Supplementary Table 3). With the cut-off values obtained with the highest Youden Index (sum of sensitivity and specificity minus one) in the ROC analyses, the PPVs were between $33.1 \%$ and $58.5 \%$, and the NPVs were between $80.9 \%$ and $89.5 \%$ (Table 4); with specificity fixed at $90 \%$, the serum makers with the highest sensitivities were Cre (36.7\%), UA (34.7\%) and BUN (34.7\%) (Supplementary Table 4). 


\section{Discussion}

Although large amount of research has been focused on preeclampsia prediction during pregnancy, very few serum prediction markers have been successfully implemented in clinical practice. With the low prevalence of preeclampsia in the general pregnant population, the application of specific laboratory test(s) would be costly to apply universally during pregnancy. In the publication for evaluating the preeclampsia predictive value of sFIt-1/PIGF by Zeisler et al., the authors narrowed down the targeting patients who presented with preeclampsia-related clinical and/or laboratory presentations (7). A similar patient recruiting strategy was adopted in our study. With a narrow focus on the subgroup of patients more likely to develop preeclampsia, medical resources may be better directed at high-risk patients; however, unlike universal screening, stratifying pregnant women based on their clinical symptoms and/or usual laboratory findings certainly requires extra effort. Whether an economic benefit exists in the overall management of preeclampsia remains a question.

According to a meta-analysis on the sFIt-1/PIGF ratio, which was recently considered one of the most promising serum markers in preeclampsia prediction, the authors found an overall sensitivity of $80 \%$, a specificity of $92 \%$, a positive likelihood ratio of 10.5 and a negative likelihood ratio of 0.22 after pooling 15 studies involving 534 cases and 19587 controls (20). A 4-week observation window along with the cutoff value of 38 was applied in the paper by Zeisler et al., which showed that the sFIt-1/PIGF ratio could accurately exclude preeclampsia occurrence in suspicious patients, with an AUC of 0.90 in the ROC analysis compared to an AUC of 0.67 in our study with follow-up until delivery (7); however, for the remaining markers included in present study, the observation window was yet to be well-defined; delivery remained the mainstream endpoint in most of the preeclampsia prediction studies $(5,21)$. The average interval between blood sampling and preeclampsia diagnosis was 7 weeks with our prospective cohort, which provided important clinical evidence for future validation studies. Interestingly, with the previously reported cut-off value of 38 for the sFIt-1/PIGF ratio and 4-week observation window, only 15 recruited subjects developed preeclampsia in our study, which was $30.5 \%(15 / 49)$ of the total preeclampsiapositive patients (Supplementary Table 5). Moreover, the NPV (94.4\%) was close to that previously reported (7); the sensitivity (40.0\%), specificity (83.4\%) and PPV (16.7\%) were much lower with our cohort (Supplementary Table 5), suggesting that ethnicity may be a confounding factor for the application of the sFlt-1/PIGF ratio and the cut-off value needs to be further optimized for Chinese populations before clinical implementation.

The hemostatic factors such as TM and tPAI-C were found to be related with the incidence and severity of PE decades ago (22). In preeclampsia patients, significant endothelial disturbance and procoagulant potential, along with aberrant expression of these hemostatic factors, were reported in previous studies $(14,15)$; however, whether they could be useful in preeclampsia prediction has yet to be investigated. With our cohort, no difference was observed between the preeclampsia positive and negative groups, indicating their limited values in preeclampsia prediction (Table 2). 
The excessive activation and poor regulation of the complement system at the maternal-fetal interface contributes to the development of preeclampsia (23). More importantly, a recent study by Jia et al. showed that the complement factors $\mathrm{C} 1 \mathrm{q}, \mathrm{B}$ and $\mathrm{H}$ were able to diagnose early-onset severe preeclampsia with AUCs of $0.81,0.74$ and 0.68 , respectively. To further evaluate their potential utility in preeclampsia prediction, the circulating levels were measured in the present study. No significant difference was found between the preeclampsia-positive and preeclampsia-negative groups (Table 2).

The two glycoproteins that were included in our testing panel, GlyFn and PAPP-A2, have been widely studied in preeclampsia. As an abundant protein with a wide spectrum of functions, serum GlyFn was found to be highly elevated during both the early and late pregnancies of the preeclampsia patients (13, 24). More interestingly, in a 2020 study by Huhn et al. (8), the GlyFn level in a prospective cohort identified with preeclampsia-specific high-risk factors was reported to show satisfactory preeclampsia prediction with an AUC of 0.94 in the ROC analysis. In our study, GlyFn was also increased, although not significantly, in the patient group that developed preeclampsia. This apparent discrepancy may be introduced by differences in the sample size and patient recruiting criteria of the two studies. In addition, a point-of-care testing system that employed fluorescently labeled fibronectin polyclonal antibodies in testing strips was used in Huhn's study, compared with the traditional ELISA assay applied with our serum samples. This distinction may further lead to the differential performance of the GlyFn level in preeclampsia prediction. The glycoprotein PAPP-A2, involved in cleaving insulin-like growth factor binding protein in the placenta, was found to be helpful in diagnosing (12) and predicting preeclampsia (8). In our study, the PAPP-A2/PIGF ratio ( $p=0.003)$ was found to be a better marker than PAPP-A2 ( $p=0.032)$ alone (Table 2), with an adjusted AUC of 0.72 (Table 3). Interestingly, PAPP-A protein, which has similar biological functions as PAPP-A2 and was a more extensively studied marker for aneuploidies and preeclampsia prediction, was found to be decreased in most of the previous preeclampsia studies (25).

As one of the essential criteria for the diagnosis of preeclampsia (6), proteinuria itself was not a sufficient predictor for the occurrence or the adverse outcomes of preeclampsia (26). Conversely, the common renal function tests such as BUN, Cre, UA and Cysc were shown to be potentially valuable in preeclampsia diagnosis and prediction. For example, the BUN (27) and BUN/Cre ratio (28) were both found increased in the preeclampsia patients compared with normal controls. Cysc, the alternative test of Cre used in glomerular filtration rate estimation, was found to be elevated in preeclampsia patients (29) and was able to predict preeclampsia in combination with neutrophil gelatinase-associated lipocalin (AUC=0.88) (30). Moreover, Cysc was reported as a predictor of preterm labor in severe preeclampsia patients, although the physiological increase of Cysc during pregnancy may pose an additional confounding factor in its clinical evaluation (31). In a prospective study with a relatively large cohort $(n=9522)$ by Rezk et al., the serum UA level during the second trimester was found to be a useful preeclampsia predictor for women at moderate or low risk (32). More interestingly, an elevated UA level was later reported to be a risk factor for women with gestational hypertension to develop preeclampsia and deliver small-for-gestational-age infants (33). We observed similar findings in which all the renal markers included (BUN, Cre, UA and Cysc) were significantly increased in the patients who developed preeclampsia before delivery. Of them, UA was 
the most promising predictor with the greatest AUCs $(0.73$ and 0.77 , before and after adjustment, respectively) in the ROC analyses (Figure 2 and Table 3 ).

There are a few limitations in this study. First, in the prospective studies of preeclampsia after $20 \mathrm{GWs}$ with patients suspected for preeclampsia development, there were no widely accepted or universal inclusion/exclusion criteria. For instance, the BP cut-off values used by Huhn for enrollment were 140/90 $\mathrm{mmHg}$ (systolic/diastolic). However, the BP elevation standards were not clearly specified in Zeisler's study (7); our clinician team decided to take a more cautionary move and had used $120 / 80 \mathrm{mmHg}$ as the cut-offs. Moreover, the patients on anti-hypertensive treatment were excluded in both Zeisler's (7) and our studies, but were allowed in Huhn's cohort (8). The use of anti-hypertensive medicine could have interfered the pathological development of preeclampsia and potentially compromise the predictive efficacy of the serum markers. Second, a longer period of time from blood sampling to preeclampsia diagnosis (average 7 weeks in present study) was used compared to other studies with prediction of imminent preeclampsia that took place in 4 weeks or less $(7,8)$ after sampling. Although using delivery as the endpoint in our study seemed reasonable since the appropriate observation window was not welldefined, the elongated study period may decrease the sensitivities or detection rates of the serum markers. More specifically, in this scenario, we would anticipate an increase of false negative samples that would have been determined as true negatives if shorter observation window (such as 4 weeks) was used. With these two limitations combined, the sensitivities for sFIt-1/PIGF ratio and other selected markers (BUN, Cre, UA, Cysc, GlyFn/PIGF and PAPP-A2/PIGF) were only between 25-36\% with a specificity of $90 \%$ (Supplementary Table 4), compared with the overall sensitivities of $>80 \%$ in the studies with similar prospective cohort $(7,8,20)$.

In conclusion, in a prospective cohort suspected of preeclampsia development, the angiogenic modulators sFlt-1 and PIGF; the renal function markers BUN, Cre, UA, and Cysc; and the glycoprotein PAPP-A2 were significantly altered between the two groups. However, with not-well-defined patient recruitment/excluding criteria and observation window, the clinical utilities of the serum biomarkers in preeclampsia prediction are limited based on the data summarized in present study. Future studies with independent cohorts of larger sizes are warranted to further reveal the values of these serum markers in preeclampsia prediction.

\section{Abbreviations}

sFlt-1, soluble fms-like tyrosine kinase 1; PIGF, placental growth factor; PAPP-A2, pregnancy-associated plasma protein-A2; GlyFn, glycosylated fibronectin; TM, thrombomodulin; tPAl-C, tissue plasminogen activator inhibitor complex; UA, uric acid; BUN, blood urea nitrogen; Cre, creatinine; Cysc, cystatin C.

\section{Declarations}

\section{Ethics approval and consent to participate}


This study was approved by the Ethics Committee of Beijing Obstetrics and Gynecology Hospital, Capital Medical University (approval number: 2017-KY-078-01). Verbal consents from the participants were required as no clinical intervention was involved, which was approved by our institute's ethical committee.

\section{Consent for publication}

Not applicable.

\section{Availability of data and materials}

The baseline characteristics of the recruited subjects are provided as Supplementary Table 1. Alternatively, Supplementary Table 1 is available in the Open Science Framework Repository (www.osf.io, DOI 10.17605/OSF.IO/JB5A7). According to the patients' verbal consents, the raw testing results are only available from the corresponding author on reasonable request.

\section{Competing interests}

The authors declare that they have no conflict of interest.

\section{Funding}

This work was supported by the Beijing Municipal Administration of Hospitals Incubating Program (No. PX2020060) and the National Natural Science Foundation of China (No. 81702057). The funding body did not take part in the design of the study, the collection, analysis and interpretation of the data, or manuscript writing.

\section{Authors' contributions}

All authors have certified the author list and the contribution description. All authors have read and approved the submitted manuscript and any substantially modified version of the manuscript. Contribution to work: J.W., H.H., X.L., S.Z., Y.Z., J.Z., Z.Z., Y.Z., J.Z. and Z.C. were involved in study conception and design and patient recruitment; L.C., C.Z., X.X. were involved in performing the experiments, and data acquisition, analysis and interpretation; H.H. and Z.C. drafted the article and critically reviewed and approved the final article; Z.J., Y.D., J.L., and Y.L. contributed to the statistical analysis and figure preparation.

\section{Acknowledgements}

The sFlt-1 and PIGF reagents were kindly provided by Beijing United JoinScience Medical Technology Co, Ltd., China. The TM and tPAI-C reagents were kindly provided by Beijing Lihaiweida Trading Co., Ltd, China. The complement component $\mathrm{C} 1 \mathrm{q}$, complement factors $\mathrm{B}$ and $\mathrm{H}$ reagents were kindly provided by Shanghai Beijia Biochemical Reagent Co. Ltd, China. The PAPP-A2 and GlyFn reagents were kindly provided by Guangzhou Kangrun Biotech Co. Ltd., Guangdong, China. 


\section{References}

1. Abalos E, Cuesta C, Grosso AL, Chou D, Say L. Global and regional estimates of preeclampsia and eclampsia: a systematic review. Eur J Obstet Gynecol Reprod Biol. 2013;170(1):1-7.

2. Phipps EA, Thadhani R, Benzing T, Karumanchi SA. Pre-eclampsia: pathogenesis, novel diagnostics and therapies. Nat Rev Nephrol. 2019;15(5):275-89.

3. Henderson JT, Thompson JH, Burda BU, Cantor A. Preeclampsia Screening: Evidence Report and Systematic Review for the US Preventive Services Task Force. JAMA. 2017;317(16):1668-83.

4. Correa PJ, Palmeiro Y, Soto MJ, Ugarte C, Illanes SE. Etiopathogenesis, prediction, and prevention of preeclampsia. Hypertens Pregnancy. 2016;35(3):280-94.

5. De Kat AC, Hirst J, Woodward M, Kennedy S, Peters SA. Prediction models for preeclampsia: A systematic review. Pregnancy Hypertens. 2019;16:48-66.

6. ACOG Practice Bulletin No. 202 Summary: Gestational Hypertension and Preeclampsia. Obstet Gynecol. 2019;133(1):211-4.

7. Zeisler H, Llurba E, Chantraine F, Vatish M, Staff AC, Sennstrom M, et al. Predictive Value of the sFlt1:PIGF Ratio in Women with Suspected Preeclampsia. N Engl J Med. 2016;374(1):13-22.

8. Huhn EA, Hoffmann I, Martinez De Tejada B, Lange S, Sage KM, Roberts CT, et al. Maternal serum glycosylated fibronectin as a short-term predictor of preeclampsia: a prospective cohort study. BMC Pregnancy Childbirth. 2020;20(1):128.

9. Herraiz I, Llurba E, Verlohren S, Galindo A, Spanish Group for the Study of Angiogenic Markers in P. Update on the Diagnosis and Prognosis of Preeclampsia with the Aid of the sFlt-1/ PIGF Ratio in Singleton Pregnancies. Fetal Diagn Ther. 2018;43(2):81-9.

10. Ng QJ, Han JY, Saffari SE, Yeo GS, Chern BSM, Tan KH. Longitudinal circulating placental growth factor (PIGF) and soluble FMS-like tyrosine kinase-1 (sFlt-1) concentrations during pregnancy in Asian women: a prospective cohort study. BMJ Open. 2019;9(5):e028321.

11. Lou WZ, Jiang F, Hu J, Chen XX, Song YN, Zhou XY, et al. Maternal Serum Angiogenic Factor sFlt-1 to PIGF Ratio in Preeclampsia: A Useful Marker for Differential Diagnosis and Prognosis Evaluation in Chinese Women. Dis Markers. 2019;2019:6270187.

12. Nishizawa H, Pryor-Koishi K, Suzuki M, Kato T, Kogo H, Sekiya T, et al. Increased levels of pregnancyassociated plasma protein-A2 in the serum of pre-eclamptic patients. Mol Hum Reprod. 2008;14(10):595-602.

13. Rasanen J, Quinn MJ, Laurie A, Bean E, Roberts CT, Jr., Nagalla SR, et al. Maternal serum glycosylated fibronectin as a point-of-care biomarker for assessment of preeclampsia. Am J Obstet Gynecol. 2015;212(1):82.e1-9.

14. Rousseau A, Favier R, Van Dreden P. Elevated circulating soluble thrombomodulin activity, tissue factor activity and circulating procoagulant phospholipids: new and useful markers for preeclampsia? Eur J Obstet Gynecol Reprod Biol. 2009;146(1):46-9. 
15. Belo L, Santos-Silva A, Rumley A, Lowe G, Pereira-Leite L, Quintanilha A, et al. Elevated tissue plasminogen activator as a potential marker of endothelial dysfunction in pre-eclampsia: correlation with proteinuria. BJOG. 2002;109(11):1250-5.

16. Jia K, Ma L, Wu S, Yang W. Serum Levels of Complement Factors C1q, Bb, and H in Normal Pregnancy and Severe Pre-Eclampsia. Med Sci Monit. 2019;25:7087-93.

17. Muller-Deile J, Schiffer M. Preeclampsia from a renal point of view: Insides into disease models, biomarkers and therapy. World J Nephrol. 2014;3(4):169-81.

18. Chen L, Liu J, Shi L, Song Y, Song Y, Gao Y, et al. Seasonal influence on TORCH infection and analysis of multi-positive samples with indirect immunofluorescence assay. J Clin Lab Anal. 2019;33(4):e22828.

19. DeLong ER, DeLong DM, Clarke-Pearson DL. Comparing the areas under two or more correlated receiver operating characteristic curves: a nonparametric approach. Biometrics 1988; 44: 837-845.

20. Agrawal S, Cerdeira AS, Redman C, Vatish M. Meta-Analysis and Systematic Review to Assess the Role of Soluble FMS-Like Tyrosine Kinase-1 and Placenta Growth Factor Ratio in Prediction of Preeclampsia: The SaPPPhirE Study. Hypertension. 2018;71(2):306-16.

21. Mosimann B, Amylidi-Mohr SK, Surbek D, Raio L. First Trimester Screening for Preeclampsia - a Systematic Review. Hypertens Pregnancy. 2020;39(1):1-11.

22. Halligan A, Bonnar J, Sheppard B, Darling M, Walshe J. Haemostatic, fibrinolytic and endothelial variables in normal pregnancies and pre-eclampsia. Br J Obstet Gynaecol. 1994;101(6):488-92.

23. Lokki Al, Heikkinen-Eloranta J, Jarva H, Saisto T, Lokki ML, Laivuori $H$, et al. Complement activation and regulation in preeclamptic placenta. Front Immunol. 2014;5:312.

24. Gredmark T, Bergman B, Hellstrom L. Total fibronectin in maternal plasma as a predictor for preeclampsia. Gynecol Obstet Invest. 1999;47(2):89-94.

25. Zhang J, Han L, Li W, Chen Q, Lei J, Long M, et al. Early prediction of preeclampsia and small-forgestational-age via multi-marker model in Chinese pregnancies: a prospective screening study. BMC Pregnancy Childbirth. 2019;19(1):304.

26. Bouzari Z, Javadiankutenai M, Darzi A, Barat S. Does proteinura in preeclampsia have enough value to predict pregnancy outcome? Clin Exp Obstet Gynecol. 2014;41(2):163-8.

27. Tokmak A, Guney G, Aksoy RT, Guzel Al, Topcu HO, Kececioglu TS, et al. May maternal anti-mullerian hormone levels predict adverse maternal and perinatal outcomes in preeclampsia? J Matern Fetal Neonatal Med. 2015;28(12):1451-6.

28. Paçarizi H, Begolli L, Lulaj S, Gafurri Z. Blood urea nitrogen/creatinine index is a predictor of prerenal damage in preeclampsia. Journal of Health Sciences. 2012;2(1):61-5.

29. Niraula A, Lamsal M, Baral N, Majhi S, Khan SA, Basnet P, et al. Cystatin-C as a Marker for Renal Impairment in Preeclampsia. J Biomark. 2017;2017:7406959.

30. Zhang HB, Fan JM, Zhu LL, Yuan XH, Shen XW. Combination of NGAL and Cystatin C for Prediction of Preeclampsia at 10-14 Weeks of Gestation. Clin Lab. 2019;65(5). 
31. Wattanavaekin $\mathrm{K}$, Kitporntheranunt M, Kreepala C. Cystatin $\mathrm{C}$ as a novel predictor of preterm labor in severe preeclampsia. Kidney Res Clin Pract. 2018;37(4):338-46.

32. Rezk M, Gaber W, Shaheen A, Nofal A, Emara M, Gamal A, et al. First versus second trimester mean platelet volume and uric acid for prediction of preeclampsia in women at moderate and low risk. Hypertens Pregnancy. 2018;37(3):111-7.

33. Zhao X, Frempong ST, Duan T. Uric acid levels in gestational hypertensive women predict preeclampsia and outcome of small-for-gestational-age infants. J Matern Fetal Neonatal Med. 2019:1-7.

\section{Tables}

Table 1 Demographic data for the recruited subjects

\begin{tabular}{|c|c|c|c|c|}
\hline & & $\begin{array}{c}\text { Preeclampsia-negative } \\
(\mathrm{n}=147)\end{array}$ & $\begin{array}{c}\text { Preeclampsia-positive } \\
(\mathrm{n}=49)\end{array}$ & $\begin{array}{c}\mathrm{p} \\
\text { value }\end{array}$ \\
\hline $\mathrm{Age}^{\mathrm{a}}$ & years & $33(29-36)$ & $34(31-37)$ & 0.066 \\
\hline Prepregnancy BMI ${ }^{\mathrm{a}}$ & $\mathrm{kg} / \mathrm{m} 2$ & $23.6(21.2-25.9)$ & $23.2(20.7-28.1)$ & 0.915 \\
\hline Sampling $\mathrm{GW}^{\mathrm{a}}$ & weeks & $29(24-33)$ & $30(25-32)$ & 0.702 \\
\hline $\begin{array}{r}\text { Preeclampsia diagnosis } \\
\text { GW }^{\mathrm{a}}\end{array}$ & weeks & Not applicable & $37(34-38)$ & \\
\hline \multirow[t]{2}{*}{ Gravidity $^{\mathrm{b}}$} & $\geq 3$ & $67.8 \%(99)$ & $59.2 \%(29)$ & 0.271 \\
\hline & $<3$ & $32.2 \%(47)$ & $40.8 \%(20)$ & \\
\hline \multirow[t]{2}{*}{ Parity ${ }^{b}$} & 0 & $74.0 \%(108)$ & $61.2 \%(30)$ & 0.090 \\
\hline & $\geq 1$ & $26.0 \%(38)$ & $38.8 \%(19)$ & \\
\hline \multirow[t]{2}{*}{$\begin{array}{r}\text { Underlying chronic } \\
\text { disease }^{\mathrm{b}}\end{array}$} & No & $84.4 \%(124)$ & $67.3 \%(33)$ & 0.010 \\
\hline & Yes & $15.6 \%(23)$ & $32.7 \%(16)$ & \\
\hline Hypertension & & $6.8 \%(10)$ & $26.5 \%(13)$ & \\
\hline $\begin{array}{l}\text { Hypothyroidism or } \\
\text { hyperthyroidism }\end{array}$ & & $2.7 \%(4)$ & $8.2 \%(4)$ & \\
\hline $\begin{array}{r}\text { Gestational diabetes } \\
\text { mellitus }\end{array}$ & & $3.4 \%(5)$ & 0 & \\
\hline Polycystic ovary syndrome & & $2.7 \%(4)$ & 0 & \\
\hline $\begin{array}{r}\text { Antiphospholipid } \\
\text { syndrome }\end{array}$ & & $0.7 \%(1)$ & 0 & \\
\hline
\end{tabular}

a: Age, prepregnancy BMI, sampling GW (gestational week), preeclampsia diagnosis GW were presented as median (25th-75th percentile).

$\mathrm{b}$ : The underlying chronic diseases that may be considered as risk factors for preeclampsia development were presented as percentages. The number in the parentheses after percentage figures indicated the number of the subjects that had the corresponding underlying chronic disease when they were enrolled. 
Table 2 Comparison of serum predictors in the preeclampsia-positive and preeclampsianegative groups

\begin{tabular}{cccc}
\hline & Preeclampsia-negative $^{\mathrm{a}}(\mathrm{n}=147)$ & Preeclampsia-positive $^{\mathrm{a}}(\mathrm{n}=49)$ & $\mathrm{p}$ value \\
\hline sFlt-1 $(\mathrm{pg} / \mathrm{ml})$ & $2036(1548-3113)$ & $2814(1785-4800)$ & 0.007 \\
\hline PlGF $(\mathrm{pg} / \mathrm{ml})$ & $301.4(135.7-511.9)$ & $209.1(69.5-293.3)$ & 0.004 \\
$\mathrm{sFlt}-1 / \mathrm{PlGF}$ & $6.8(3.6-21.7)$ & $13.3(6.8-65.0)$ & $<0.001$ \\
$\mathrm{TM}(\mathrm{IU} / \mathrm{ml})$ & $9.9(8.7-10.2)$ & $10.7(8.8-12.3$ & 0.405 \\
\hline $\mathrm{tPAI}-\mathrm{C}(\mathrm{ng} / \mathrm{ml})$ & $5.9(4.5-7.3)$ & $5.4(3.7-6.8)$ & 0.154 \\
\hline $\mathrm{BUN}(\mathrm{mmol} / \mathrm{L})$ & $2.8(2.4-3.4)$ & $3.3(2.5-4.3)$ & 0.009 \\
\hline $\mathrm{Cre}(\mathrm{\mu mol} / \mathrm{L})$ & $40.8(36.8-44.8)$ & $45.4(38.1-51.1)$ & 0.006 \\
\hline $\mathrm{UA}(\mu \mathrm{mol} / \mathrm{L})$ & $232.3(201.5-280.1)$ & $295.7(233.2-336.0)$ & $<0.001$ \\
\hline Cysc $(\mathrm{mg} / \mathrm{ml})$ & $0.9(0.8-1.1)$ & $1.1(0.9-1.4)$ & 0.001 \\
\hline $\mathrm{C} 1 \mathrm{q}(\mathrm{mg} / \mathrm{L})$ & $204.0(177.0-228.0)$ & $194.0(170.0-226.0)$ & 0.346 \\
\hline B facstor $(\mathrm{mg} / \mathrm{L})$ & $333.0(308.0-365.0)$ & $353.5(328.5-369.3)$ & 0.036 \\
\hline $\mathrm{H}$ factor $(\mathrm{mg} / \mathrm{L})$ & $404.0(372.0-429.0)$ & $397.5(358.0-436.8)$ & 0.664 \\
\hline GlyFn $(\mathrm{mg} / \mathrm{ml})$ & $259.1(220.1-323.3)$ & $285.4(227.1-421.9)$ & 0.061 \\
\hline PAPP-A2 $(\mathrm{mg} / \mathrm{ml})$ & $52.9(31.4-103.7)$ & $91.8(38.3-192.7)$ & 0.032 \\
\hline GlyFn/PlGF & $0.9(0.5-2.3)$ & $1.5(0.8-6.0)$ & 0.002 \\
\hline PAPP-A2/PlGF & $0.2(0.1-0.7)$ & $0.5(0.1-2.3)$ & 0.003 \\
\hline
\end{tabular}

a: presented as median (25th-75th percentile).

Table 3 Comparison of AUCs before and after adjusted for demographic data of recruited subjects

\begin{tabular}{cccc}
\hline & Unadjusted AUC $^{\mathrm{a}}\left(95 \% \mathrm{CI}^{\mathrm{b}}\right)$ & Adjusted $^{\mathrm{c}} \mathrm{AUC}(95 \% \mathrm{CI})$ & $\mathrm{p} \mathrm{value}^{\mathrm{d}}$ \\
\hline sFlt-1/PlGF & $0.67(0.59-0.73)$ & $0.70(0.63-0.77)$ & 0.457 \\
\hline BUN $(\mathrm{mmol} / \mathrm{L})$ & $0.63(0.56-0.70)$ & $0.70(0.62-0.76)$ & 0.050 \\
\hline Cre $(\mu \mathrm{mol} / \mathrm{L})$ & $0.63(0.57-0.72)$ & $0.73(0.66-0.79)$ & 0.057 \\
\hline UA $(\mu \mathrm{mol} / \mathrm{L})$ & $0.73(0.66-0.79)$ & $0.77(0.70-0.83)$ & 0.104 \\
\hline Cysc $(\mathrm{mg} / \mathrm{ml})$ & $0.66(0.59-0.73)$ & $0.70(0.63-0.76)$ & 0.312 \\
\hline GlyFn/PlGF & $0.65(0.57-0.71)$ & $0.70(0.63-0.76)$ & 0.225 \\
\hline PAPP-A2/PlGF & $0.64(0.57-0.71)$ & $0.72(0.65-0.79)$ & 0.058 \\
\hline
\end{tabular}

a: AUC, area under the curves; b: CI, confidence interval;

c: adjusted for age, prepregnancy BMI, parity and underlying chronic disease;

$\mathrm{d}$ : comparison $\mathrm{p}$ valued before and after adjusted AUC. 
Table 4 The performances of serum biomarkers in predicting preeclampsia

\begin{tabular}{cccc} 
& Cut-off value & PPV $^{\mathrm{a}}(\%)$ & NPV $^{\mathrm{b}}(\%)$ \\
\hline sFlt-1/PlGF & 5.6 & 34.0 & 89.5 \\
BUN $(\mathrm{mmol} / \mathrm{L})$ & 3.9 & 58.5 & 80.9 \\
Cre $(\mu \mathrm{mol} / \mathrm{L})$ & 48.0 & 53.5 & 82.7 \\
$\mathrm{UA}(\mu \mathrm{mol} / \mathrm{L})$ & 280.7 & 46.0 & 85.6 \\
\hline Cysc $(\mathrm{mg} / \mathrm{ml})$ & 1.0 & 37.1 & 83.1 \\
\hline GlyFn/PlGF & 0.7 & 33.1 & 88.0 \\
\hline PAPP-A2/PlGF & 1.0 & 48.0 & 81.5 \\
\hline
\end{tabular}

a: positive predictive value. b: negative predictive value.

\section{Figures}

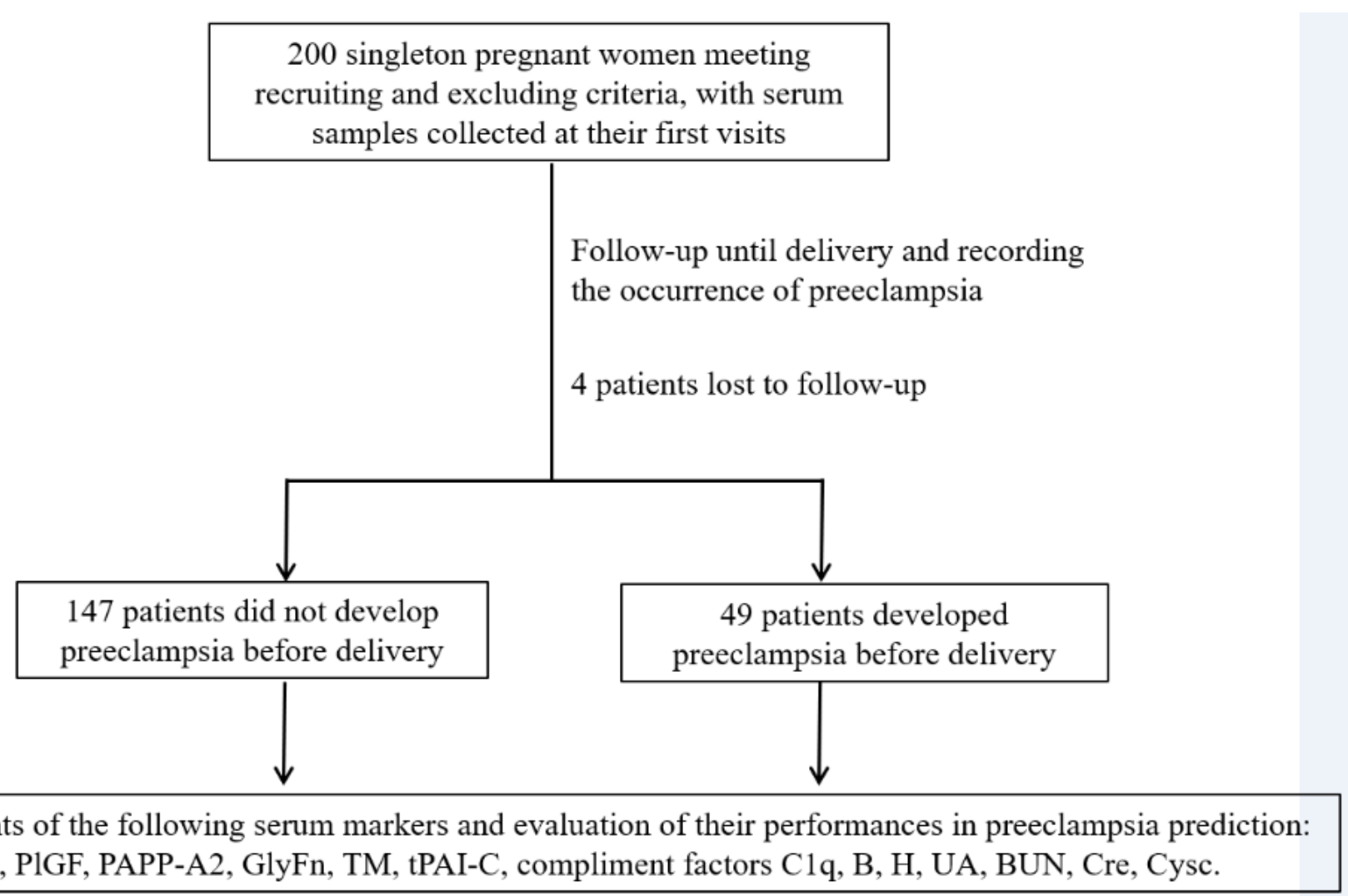

\section{Figure 1}

Schematic diagram depicting patient recruitment and study design. Abbreviations: sFlt-1, soluble fms-like tyrosine kinase 1; PIGF, placental growth factor; PAPP-A2, pregnancy-associated plasma protein-A2; GlyFn, glycosylated fibronectin; TM, thrombomodulin; tPAI-C, tissue plasminogen activator inhibitor complex; UA, uric acid; BUN, blood urea nitrogen; Cre, creatinine; Cysc, cystatin C. 

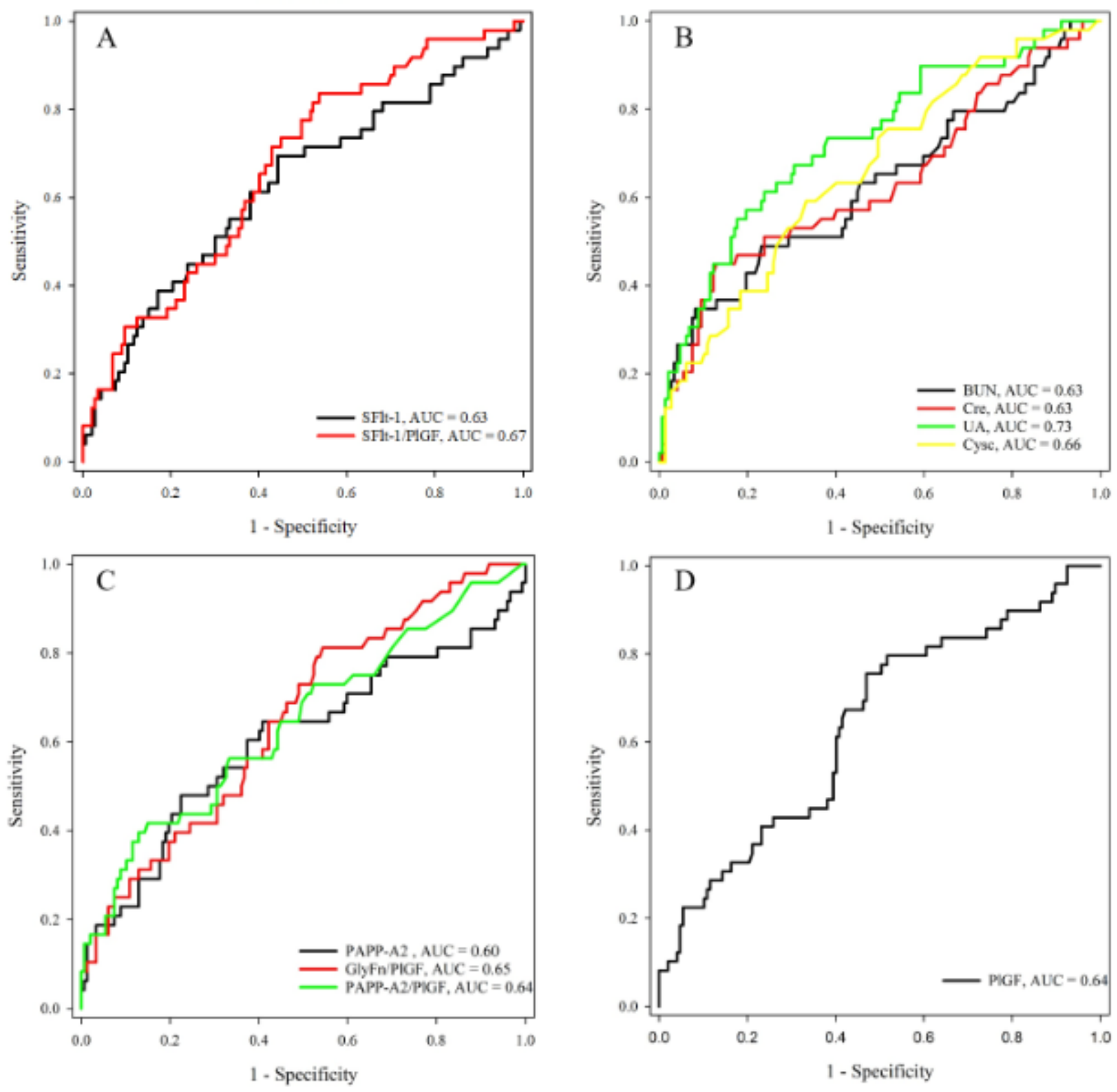

Figure 2

ROC analyses of the serum markers for $\mathrm{PE}$ prediction in the prospective cohort with $\mathrm{PE}$-related clinical or laboratory presentations. A. ROC analyses for sFlt-1 (AUC=0.63) and sFIt-1/PIGF (AUC=0.67); B. BUN $(A \cup C=0.63)$, Cre (AUC=0.63), UA (AUC=0.73) and Cysc $(A U C=0.66)$;. PAPP-A2 (AUC=0.60), GlyFn/PIGF $(A \cup C=0.65)$ and PAPP-A2/PIGF (AUC=0.64); D. PIGF (AUC=0.64).

\section{Supplementary Files}

This is a list of supplementary files associated with this preprint. Click to download.

- STROBEchecklistcohortPEmarkers.doc

- SupplementaryTablesrevise.xlsx 